\title{
Demarcated redness associated with increased vascular density/size: a useful marker of flat-type dysplasia in patients with ulcerative colitis
}

\section{(ㄷ)(1) $(2) \Theta$}

Authors

Akiyoshi Ikebata ${ }^{1,2}$, Masayuki Shimoda ${ }^{1}$, Koji Okabayashi ${ }^{2}$, Toshio Uraoka ${ }^{3,4}$, Tadateru Maehata ${ }^{3}$, Shinya Sugimoto $^{5}$, Makoto Mutaguchi ${ }^{5}$, Makoto Naganuma ${ }^{5}$, Kaori Kameyama ${ }^{6}$, Naohisa Yahagi ${ }^{3}$, Takanori Kanai ${ }^{5}$, Yuko Kitagawa², Yae Kanai', Yasushi Iwao7

Institutions

1 Department of Pathology, Keio University School of Medicine, Tokyo, Japan

2 Department of Surgery, Keio University School of Medicine, Tokyo, Japan

3 Division of Research and Development for Minimally Invasive Treatment, Cancer Center, Keio University School of Medicine, Tokyo, Japan

4 Department of Gastroenterology and Hepatology, Gunma University Graduate School of Medicine, Maebashi, Japan

5 Division of Gastroenterology and Hepatology, Department of Internal Medicine, Keio University School of Medicine, Tokyo, Japan

6 Division of Diagnostic Pathology, Keio University School of Medicine, Tokyo, Japan

7 Center for Preventive Medicine, Keio University School of Medicine, Tokyo, Japan

submitted 20.5.2020

accepted after revision 27.11.2020

Bibliography

Endosc Int Open 2021; 09: E552-E561

DOI 10.1055/a-1352-2709

ISSN 2364-3722

(C) 2021. The Author(s).

This is an open access article published by Thieme under the terms of the Creative Commons Attribution-NonDerivative-NonCommercial License, permitting copying and reproduction so long as the original work is given appropriate credit. Contents may not be used for commercial purposes, or adapted, remixed, transformed or built upon. (https://creativecommons.org/licenses/by-nc-nd/4.0/)

Georg Thieme Verlag KG, Rüdigerstraße 14,

70469 Stuttgart, Germany

Corresponding authors

Masayuki Shimoda, MD, PhD, Department of Pathology, Keio University School of Medicine, Tokyo, Japan shimoda@a2.keio.jp

Supplementary material is available under

https://doi.org/10.1055/a-1352-2709

\section{ABSTRACT}

Background and study aims Recent advances in endoscopic equipment and diagnostic techniques have improved the detection of dysplasia in the inflamed mucosa of patients with ulcerative colitis (UC). However, it remains difficult to endoscopically identify flat-type dysplasia which has been formerly recognized as invisible dysplasia.

Patients and methods In this retrospective, single-center study, we endoscopically identified 10 cases of flat-typepredominant dysplasia by targeted biopsy among 38 intramucosal dysplasia lesions from patients with UC who underwent surgical or endoscopic resection from 2007 to 2017. Their endoscopic and histological features were examined, including color changes, intramucosal vascular density/size, and vascular endothelial growth factor (VEGF) expression.

Results All flat-type-predominant dysplasias were endoscopically recognized as demarcated red-colored areas and histologically diagnosed as low- (LGDs) or high-grade dysplasias (HGDs). Immunohistochemical examination using resected specimens revealed that flat-type dysplasia was characterized by significantly increased CD34-positive vascular density (LGDs, 1.7-fold, $P<0.01$; HGDs, 2.2-fold, $P<$ 0.01 ) and size (LGDs, 1.03-fold, $P<0.01$; HGDs, 1.11-fold, $P<0.01)$ in the mucosa, compared to adjacent non-neoplastic areas. Increased numbers of vessels were observed at the base of the mucosa in LGDs, whereas HGDs contained increased/enlarged vessels throughout the mucosa. Moreover, VEGF expression was elevated in all dysplastic epithelia.

Conclusions Demarcated red-colored areas, histologically characterized by an increased vascular density/size in the mucosa, are an endoscopic sign of formerly invisible flattype dysplasia in patients with UC and should be considered for targeted biopsy. Prospective studies focusing on the mucosal color change for their early detection would be desirable in the future. 


\section{Introduction}

It is generally accepted that long-standing ulcerative colitis (UC) is associated with an increased risk of colorectal cancer $[1,2]$. The cumulative risk reaches $7.5 \%$ to $18.4 \%$ at 30 years after onset of the disease [2]; therefore, early detection of UCassociated colorectal cancer is recommended for successful management of patients with UC. Despite recent advances in endoscopic equipment, patient preparation, and diagnostic techniques, the early detection of colitis-associated cancer or dysplasia remains difficult because of the complex background of the inflamed mucosa of UC and the morphologic diversity of lesions.

The Surveillance for Colorectal Endoscopic Neoplasia Detection and Management in Inflammatory Bowel Disease Patients: International Consensus Recommendations (SCENIC) consensus statement proposes the following five types of endoscopic morphological features of colitis-associated dysplasia: pedunculated, sessile, superficial elevated, flat, and depressed lesions $[3,4]$. Using these criteria, we reported that $64.1 \%$ of highgrade dysplasia (HGD) lesions were associated with elevated areas (sessile, superficial elevated), whereas $30.8 \%$ were classified as flat lesions, which were previously recognized as invisible lesions and are rare in patients with sporadic dysplasia [5]. Notably, multiple synchronous cancers are more frequent in patients with colitis-associated cancers; these tumors typically arise from flat dysplastic lesions with a mutational burden, suggesting a field cancerization process in the context of chronic inflammation. Several guidelines have suggested colectomy for such flat-type dysplasias in patients with UC because of the higher risk of colorectal cancer and the difficulty of complete endoscopic removal of these lesions [6,7].

To prevent the development of synchronous and/or invasive colitis-associated cancers in patients with long-standing UC, it is important that endoscopists recognize the endoscopic and histological features of invisible flat-type dysplasias to facilitate their early detection. Although some flat dysplastic lesions are difficult for even experienced endoscopists to detect by highdefinition chromoendoscopy, especially in patients with severe inflammation, we recently proposed that flat dysplastic lesions are endoscopically characterized by abnormalities with a distinct border and red coloration [5]. Similar red-colored changes associated with increased intramucosal capillaries have been observed in flat early gastric cancer [8-10], suggesting that the "demarcated red-colored change" in patients with UC can serve as a diagnostic marker of flat-type dysplasia. However, it has been reported that narrow-band imaging (NBI), which allows endoscopic observation of microvessels in the mucosa, is not useful for the detection of these lesions; moreover, it remains controversial whether these color changes are an endoscopic marker of flat-type dysplasia [3]. In addition, the factors/mechanisms responsible for these color changes are unknown. In this study, we investigated the endoscopic and histological features of endoscopically detected flat-type dysplasias in patients with UC, focusing on the association between endoscopic color changes and histological microvascular changes in the mucosa using resected specimens.

\section{Patients and methods}

\section{Patients}

We enrolled 38 cases of intramucosal low-grade dysplasia (LGD), HGD, and dedifferentiated cancer such as signet-ring cell carcinoma endoscopically detected by targeted biopsy and subsequently underwent surgical or endoscopic resection at Keio University Hospital (Tokyo, Japan) from January 2007 to December 2017 [11]. The resected samples were confirmed by histopathological assessment by at least two experienced pathologists (MS and KK). Histological diagnosis was confirmed by routine hematoxylin and eosin staining, with respect to the immunohistochemical expression patterns of p53 and Ki-67, as previously described [12]. Because all the dysplastic lesions in this study showed nuclear and architectural atypia as well as strongly/diffusely positive or completely negative pattern of p53 expression, there were no discrepancies as for the diagnosis of dysplasia. However, since there were some discrepancies regarding the degree of dysplasia (low-grade vs high-grade) between two pathologists, we observed all the specimens carefully, discussed and determined the degree of the dysplasia considering the nuclear and architectural atypia, cellular differentiation and p53 staining pattern. Cases of visible LGD/HGD, in which sporadic lesions could not be ruled out, were excluded. From this cohort, we retrospectively identified ten cases of flattype-predominant LGDs/HGDs in nine patients with UC and two cases of intramucosal tumors containing HGD and signet-ring cell carcinoma components in two patients with UC. The demographic data and disease characteristics of the patients were evaluated, including age, sex, duration of disease at detection of neoplastic lesion, and clinical type and extent of the disease. The study protocol was reviewed and approved by the Ethics Committee of Keio University School of Medicine (approval no. 20180209).

\section{Definition and evaluation of the endoscopic findings}

Possible flat-type dysplastic lesions were detected based on the color change using regular white-light colonoscopy (CF-Q240I, H290Al or PCF-Q260AI, Q260AZI, H290I; Olympus, Tokyo, Japan). Identified lesions were observed following $0.2-0.4 \%$ indigo carmine dye spraying. To further determine whether these areas were dysplastic or non-neoplastic, subsequent magnifying colonoscopy (CF-H260AZI, HQ290I or PCF-Q260AZI, H290AZI; Olympus) using NBI and/or $0.05 \%$ crystal violet staining were performed to identify their lines of demarcation. The endoscopic morphology was classified in accordance with the SCENIC international consensus statement. Briefly, flat lesions were defined as tumors without elevation or depression relative to the surrounding mucosa and could be detected only by their color change. Classifications were determined retrospectively after thorough discussion by two expert endoscopists ( $\mathrm{YI}$ and $\mathrm{MN}$ ) based on the detailed information provided by reporting endoscopists. The endoscopic disease activity was assessed by trained colonoscopists in accordance with the Mayo endoscopic score. 


\section{Immunohistochemistry and staining assessment}

Four-micrometer-thick paraffin-embedded sections were subjected to immunohistochemistry using standard histological protocols $[13,14]$. Immunohistochemical staining was performed using a Bond-Max automated immunohistochemical stainer (Leica Microsystems, Milton Keynes, UK). The primary antibodies used were anti-Ki-67 (1:200 dilution; Cat. No. MIB1; Dako, Carpinteria, CA, USA), anti-p53 (1:2000 dilution; Cat. No. DO-7; Dako), anti-CD34 (1:100 dilution; Cat. No. QEBnd10; Dako) and anti-VEGF (1:400 dilution; Cat. No.JH121; Millipore, Billerica, MA, USA). CD34-positive vessels with lumen structures in the mucosa were assessed by Al and MS to evaluate microvascular densities and sizes. Specifically, the numbers of vessels in mucosae were counted by observation of all fields of each specimen at $\times 200$ magnification and calculation of the microvascular density per square millimeter. The short diameter of each vessel was also measured. To map the vascular density in resected specimens, the $75^{\text {th }}$ percentile was used to discriminate a high and low density; the cut-off values of vessel density in cases \#2, \#5, and \#7 were 42.1 vessels $/ \mathrm{mm}^{2}, 48.2$ vessels $/ \mathrm{mm}^{2}$, and 38.5 vessels $/ \mathrm{mm}^{2}$, respectively. The abnormal expression of p53 was defined as strongly/diffusely positive or completely negative immunoexpression pattern. The proportion of positive neoplastic/non-neoplastic cell staining for VEGF was graded as follows: no positive cells or essentially none $(<5 \% ; 0+)$, some positive cells $(5 \%$ to $30 \% ; 1+)$, diffuse areas of positive cells ( $30 \%$ to $60 \% ; 2+$ ), and extensive areas of positive cells (>60\%; $3+$ ).

\section{Statistical analysis}

Nonparametric tests were used in this study. For the vessel evaluation at x200 magnification in neoplastic and non-neoplastic lesions, continuous variables were expressed in terms of the median (interquartile range) (Supplementary Table 3 and Supplementary Table 7). The Mann-Whitney $U$ test was used to compare categorical and continuous variables. The Kruskal-Wallis test was used to assess differences in the distributions of categorical variables. Differences with $P<0.05$ were considered statistically significant. Data were analyzed using Stata, version 11.2 (Stata Corporation, College Station, Texas, United States).

\section{Results}

\section{Clinical, endoscopic, and histological characteristics}

Among 38 intramucosal colitis-associated dysplasia/cancer lesions in patients with UC who underwent surgical or endoscopic resection, 10 flat-type-predominant dysplastic lesions in nine patients were detected by endoscopy and histologically diagnosed as LGDs and/or HGDs. The clinical and endoscopic characteristics of these nine patients with UC are shown in Supplementary Table 1 and Supplementary Table 2. All nine patients showed a duration of median relapse-remitting disease of 24 years (interquartile range, 16 to 31); their median age at the time of detection was 49 years (interquartile range, 46 to $56)$. Dysplastic lesions were mainly detected in the rectum or sigmoid colon (90\%), including six lesions in the rectum, three in the sigmoid colon, and one in the ascending colon. Endoscopically, seven lesions (cases \#1, 2, 5, 6, 8, 9-1, and 9-2) (Supplementary Table 2) were classified as flat lesions, in accordance with the SCENIC consensus statement. The others (cases \#3, 4, and 7) were categorized as sessile lesions, but also contained flat dysplastic areas that widely spread around the sessile areas. Importantly, all flat dysplastic lesions were recognized as red-colored areas in remitted colonic mucosa by white-light image and their lines of demarcation were enhanced by NBI ( $\triangleright$ Fig. 1). The histological findings of 10 lesions are shown in Supplementary Table 2. In total, four (40\%), two (20\%), and four (40\%) flat dysplastic lesions were classified as LGDs, HGDs, and combined LGDs/HGDs, respectively. All sessile lesions observed in cases \#3, \#4, and \#7 were HGDs. Endoscopic submucosal dissection or curative colectomy with lymph node resection was performed in all patients; none experienced recurrence during the observation period.

\section{Histological assessment of intramucosal vascular changes in dysplastic lesions}

Intramucosal microvascular densities/sizes in dysplasias and adjacent non-neoplastic mucosae were evaluated by immunohistochemical staining for CD34. As shown in > Fig. 2a, the number of CD34-positive vessels in p53-positive flat dysplastic mucosae were markedly increased, compared to surrounding p53negative non-neoplastic areas. Detailed mapping of vascular density by assessment of the numbers of CD34-positive vessels in neoplastic and surrounding non-neoplastic areas revealed that the CD34-positive vascular-increased area was largely restricted to the histologically confirmed dysplastic lesions ( Fig.2b). The intramucosal vascular densities in flat LGD (1.7-fold, $P<0.01)$, flat HGD $(2.2$-fold, $P<0.01)$, and sessile HGD (1.9-fold, $P<0.01$ ) lesions were significantly greater than the vascular density in surrounding non-neoplastic mucosa. The vessels in flat LGD, flat HGD, and sessile HGD areas were also significantly enlarged by 1.03-, 1.11-, and 1.15-fold, respectively, compared to non-neoplastic mucosa $(P<0.01)$ ( Fig. 3, \Fig.4a, \Fig.4b, and Supplementary Table 3). Histologically, LGDs contained p53-positive or completely negative atypical epithelia confined largely to the basal half of the glands, whereas atypical epithelia extended into the superficial (luminal) part of the gland in most HGDs [15]. To further investigate the intramucosal distribution of increased/enlarged vessels in LGDs and HGDs, we divided the mucosa into basal and luminal halves and compared the vascular densities and sizes between these halves ( $\mathbf{F i g . 4 c}$ ). As shown in $>$ Fig. 3, increased/enlarged vessels were mainly observed in the basal half in LGDs, whereas these vessels extended into the luminal half in HGDs. The ratio of the vascular density in the luminal half to the vascular density in the basal half was significantly reduced in LGD lesions, compared to non-neoplastic lesions $(\mathrm{P}<$ 0.01 ); this ratio gradually increased in flat HGD and sessile HGD lesions. The ratio of the vascular size in the luminal half to the vascular size in the basal half also tended to be lower in non-neoplastic and LGD lesions than in flat HGD and sessile

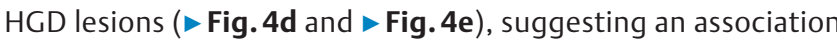




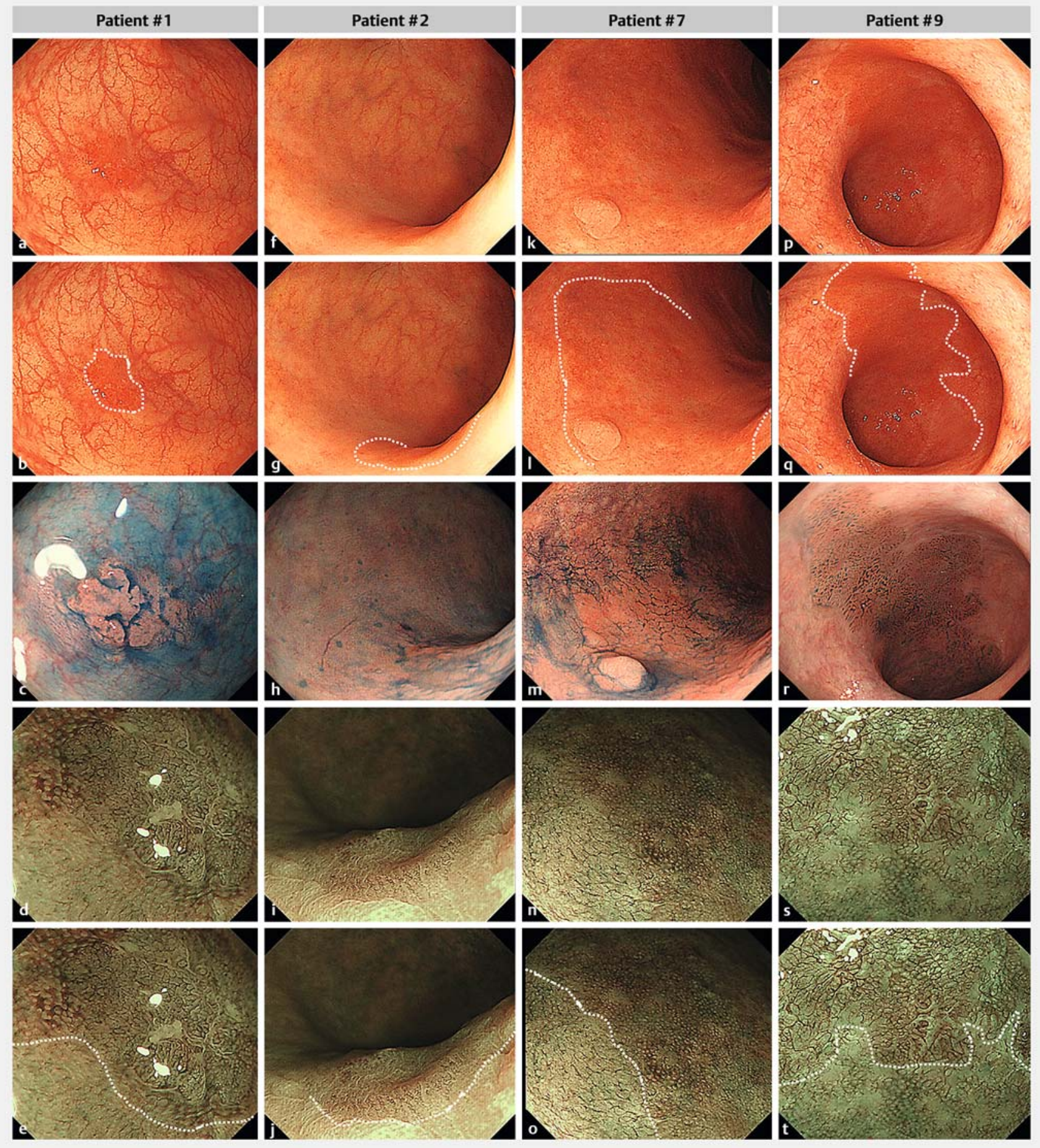

- Fig. 1 Representative endoscopic images of flat-type dysplasia in UC patients. a, b, c, d, e Flat (Patient \#1). f, g, h, i, j Flat (Patient \#2). $\mathbf{k}, \mathbf{I}, \mathbf{m}, \mathbf{n}, \mathbf{o}$ Flat + sessile (Patient \#7). p, q, r, s, t Flat lesion (Patient \#9), according to the SCENIC consensus statement. These lesions were observed on white-light imaging (WLI) (the first and second row from the top), indigo-carmine dye splaying (the third row) and magnifying narrow-band imaging (NBI) (the fourth and fifth row). Note that reddish areas on WLI were indicated by white dotted lines in the second row. The border of vascular increased areas on NBI was also demonstrated by white dotted lines in the fifth row. 


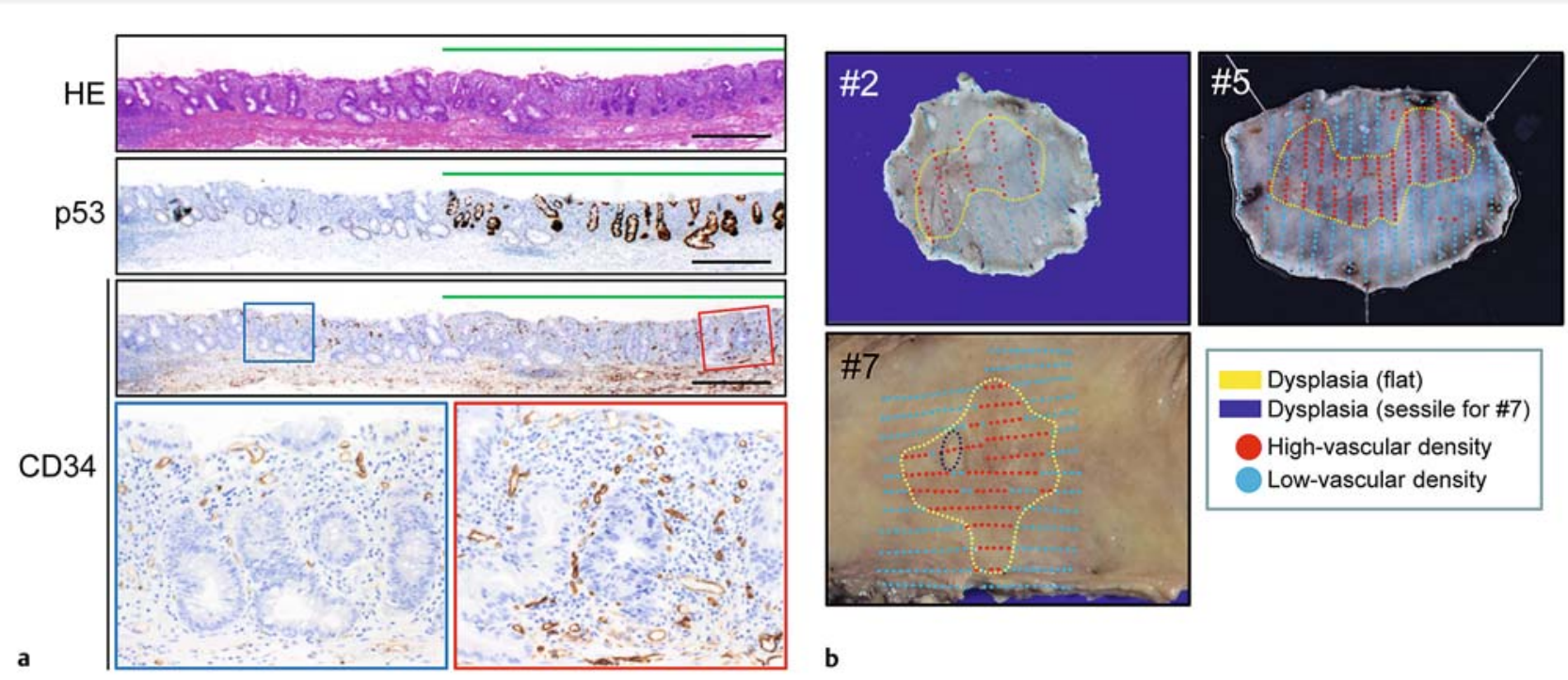

- Fig. 2 Representative histological and macroscopic images in flat-type dysplasia. a Representative histological images of flat-type high-grade dysplasia (HGD) (Case \#7). The histological findings for the resected specimen: HE staining indicating a flat-type HGD lesion (upper) and p53 immunohistochemistry (middle) indicating overexpression of p53. Note that CD34-positive vessels in the mucosa were increased in the HGD lesions compared to in the adjacent non-neoplastic lesions by CD34 immunohistochemistry (lower). The rectangle dysplastic (red line) and non-neoplastic (blue line) areas are magnified below. Scale bars, $500 \mu \mathrm{m}$. b Representative macroscopic images of resected specimens of flattype dysplasia showing mucosal vascular densities (Case \#2, \#5, and \#7). Red and blue dots indicate high- and low-density of intramucosal vessels, respectively. The $75^{\text {th }}$ percentile was used to determine high- and low-density of intramucosal vessels as described in the "Patients and methods" section. Dysplastic areas confirmed by histological assessment were lined by yellow dotted lines. Case \#7 contains a sessile lesion indicated by a black dotted line.

of stromal vascular changes with p53-positive atypical epithelial areas.

\section{Increased VEGF expression in dysplastic epithelia}

Vascular endothelial growth factor (VEGF) is a potent angiogenic factor with a key role in several pathological processes, including tumor vascularization [16]. Immunohistochemistry revealed that all LGD and HGD epithelia showed increased VEGF expression, compared to surrounding non-neoplastic epithelia (Supplementary Table 4). Moreover, the pattern of VEGF expression in dysplastic epithelia was similar to that of $\mathrm{p} 53$; namely, VEGF expression was mainly observed in the basal half of LGD glands, whereas its expression extended into the surface in HGD glands (> Fig.5a), suggesting links among p53 alteration, VEGF expression, and stromal vascular changes ( $\triangleright$ Fig. $5 b)$.

\section{Exceptional discoloration of dysplastic epithelia: intramucosal signet-ring cell carcinoma}

Although rare, colorectal signet-ring cell carcinoma is more frequent in patients with UC. In a few cases (Supplementary Table 5 and Supplementary Table 6), intramucosal signet-ring cell carcinoma areas within HGDs detected in superficial elevated or depressed dysplastic lesions were endoscopically recognized as discolored and histologically contained fewer vessels. Signet-ring cell carcinoma cells also tended to show mildly lower VEGF expression, compared to adjacent HGD lesions ( $\mathbf{F i g .}$ 6). The intramucosal vascular density in signet-ring cell carci- noma areas was significantly lower than the vascular density in surrounding HGD lesions (cases \#S1 and \#S2) (Supplementary Table 7).

\section{Discussion}

Improved early detection of flat-type dysplasias is important for prevention of colorectal cancer in patients with long-standing UC. In this study, we evaluated the endoscopic and histological features of flat-type dysplasias by histological assessment of completely resected flat-type dysplasias that had been endoscopically detected with the clue of demarcated red-colored change, focusing on stromal vascular changes. Immunohistochemistry revealed that both flat-type LGD and HGD lesions endoscopically recognized as red were characterized by increased CD34-positive microvascular density/size in the mucosa, compared to adjacent non-neoplastic areas. The number of vessels was increased at the base of the mucosa in LGDs, whereas HGDs contained increased/enlarged vessels throughout the mucosa, accompanied by increased VEGF expression in dysplastic epithelia. To the best of our knowledge, this is the first report of the endoscopic and histological features of flattype dysplasia in patients with UC, and the demarcated redness may be a useful marker for early detection of flat-type dysplasia by endoscopy.

Mucosal changes are an initial indication of flat-type gastrointestinal tumors and include redness, discoloration, and spotty bleeding. These changes can be influenced by the histologi- 


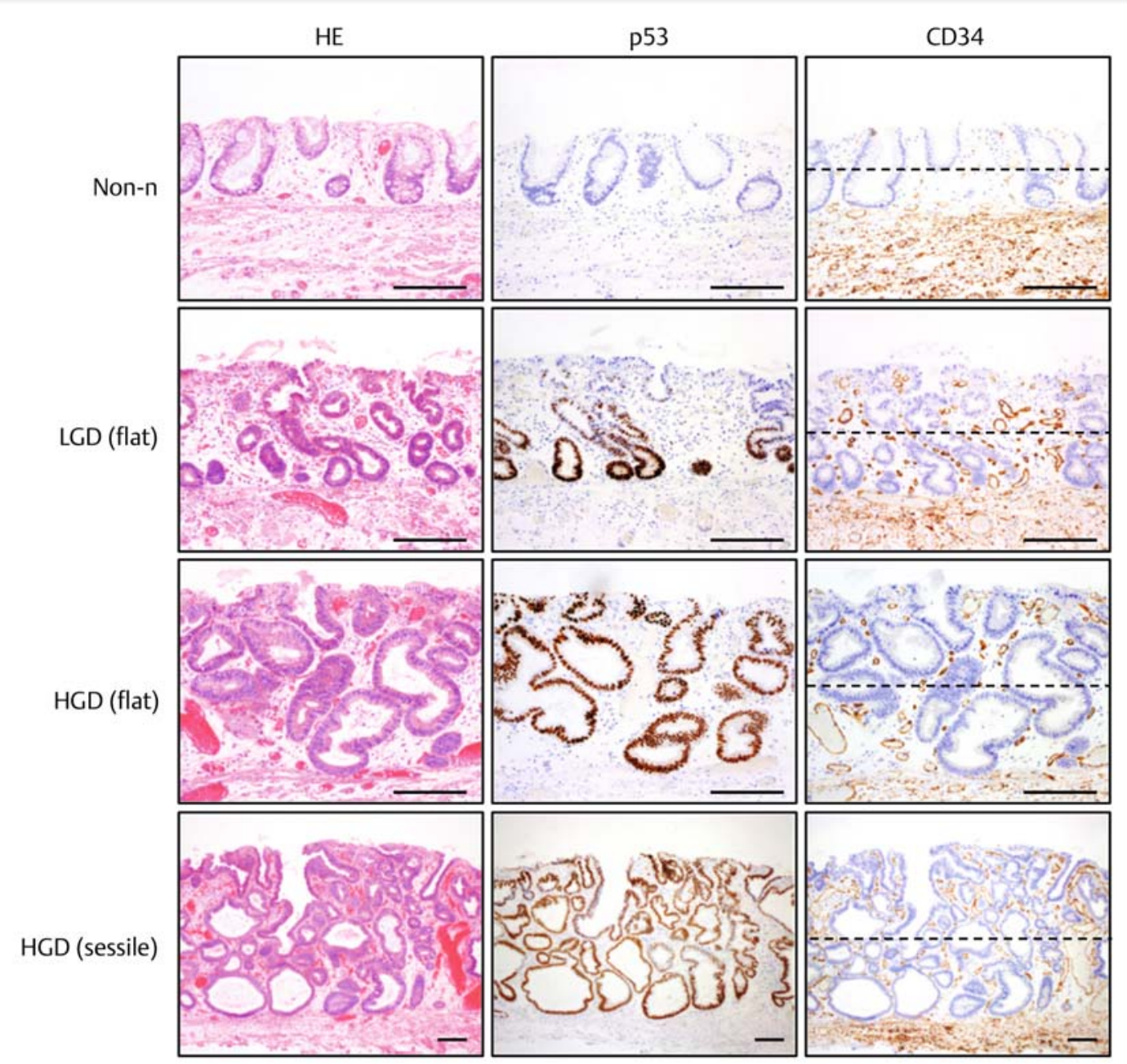

- Fig. 3 Representative histological images of non-neoplastic, flat-type low-grade dysplasia, flat-type high-grade dysplasia and sessile highgrade dysplasia. The histological findings (Case \#4): HE staining indicating non-neoplastic (Non-n), flat low-grade dysplasia (LGD), flat-type high-grade dysplasia (HGD) and sessile HGD lesions (left), p53 immunohistochemistry (middle), and CD34 staining indicating vessels in the mucosa (right). In CD34-immunostained sections, the luminal and basal half areas were divided by black dotted lines. Scale bars, $200 \mu \mathrm{m}$.

cal type of the tumor, as well as stromal changes including inflammation, edema, fibrosis, and microvascular architectures. The redness and discoloration of the mucosa, accompanied by stromal vascular alterations, are reportedly useful for early detection of invisible flat differentiated and undifferentiated gastric carcinomas, respectively [8,9]. Flat-type dysplastic lesions, which are rare in colonic sporadic dysplasia [17], are frequent in patients with UC; these lesions were formerly recognized as "invisible dysplasia." However, our randomized controlled trial revealed that targeted biopsies were comparable to random biopsies in terms of dysplasia detection by experts [18]. This study demonstrated that improvements in technique and technology have enabled visualization of these lesions, but it remains uncertain whether this visualization can be performed by both UC specialists and non-UC specialists. It is necessary to establish objective endoscopic findings that allow non-specialists to perform targeted biopsy to detect flat-type dysplasias in patients with UC, which are diagnosed with increasing frequency.

Several studies have described the endoscopic markers of UC-associated dysplasia. For example, a tortuous pattern and irregular/amorphous surface patterns are reportedly useful for detection of dysplasia in patients with UC by magnifying chromoendoscopy [19]. Recently, combined magnifying endoscopic and stereomicroscopic analyses demonstrated that neoplastic pit patterns (types III, IV, and V) and surface morphologies, such as pinecone/villi patterns, were also significantly correlated with the presence of dysplasia [20]. These magnified observations can indeed provide precise images that aid in the diagnosis of neoplastic lesions in patients with UC; however, they are not useful for routine surveillance because the magnified field of view is very small. In addition, these studies revealed that some neoplastic lesions at the bottom of glands, such as flat-type LGDs, showed a non-neoplastic pit pattern; this finding suggests limitations in the detection of flat-type 

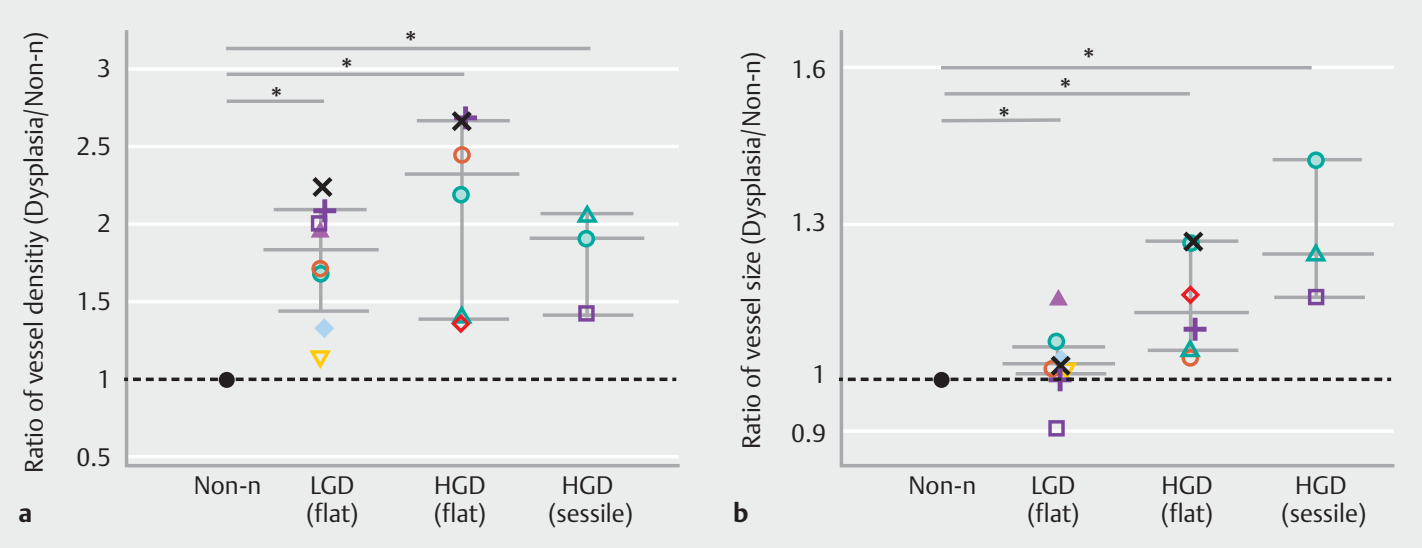

$\triangle$ Case 1

+ Case 2

$\square$ Case 3

- Case 4

$\times$ Case 5

- Case 6

$\triangle$ Case 7

$\diamond$ Case 8

Case 9-1

$\nabla$ Case 9-2
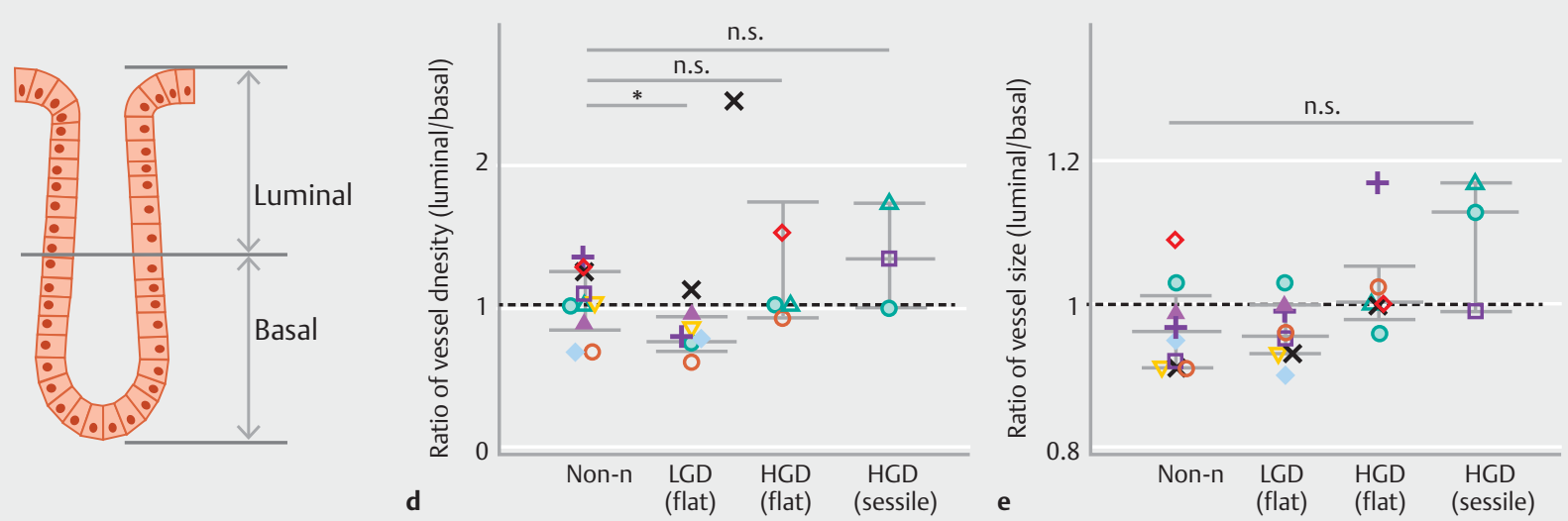

c

d

(flat) (flat) (sessile)

e

(flat) (flat) (sessile)

- Fig. 4 Evaluation of intramucosal vessel density/size in non-neoplastic, low-grade dysplasia and high-grade dysplasia. a Evaluation of intramucosal vessel density in non-neoplastic (Non-n) $(n=10)$, low-grade dysplasia (LGD) $(n=7)$, flat-type high-grade dysplasia $(H G D)(n=5)$ and sessile HGD lesions $(n=3)$. The ratio of average vessel density in LGD, flat-type HGD or sessile HGD lesions to that in Non- $n$ lesions in each case is shown. b Evaluation of intramucosal vessel size in Non- $(n=10)$, LGD $(n=7)$, flat-type HGD $(n=5)$ and sessile HGD lesions $(n=3)$. The ratio of average vessel size in LGD, flat-type HGD or sessile HGD lesions to that in Non-n lesions in each case is shown. c Schematic presentation showing the separation of colonic crypts and their surrounding stroma into the luminal and basal half areas. $\mathbf{d}$ Evaluation of vessel density in the luminal and basal half areas of Non- $(n=10)$, LGD $(n=7)$, flat-type HGD $(n=5)$ and sessile HGD lesions $(n=3)$. The ratio of average vessel density in the luminal half areas to that in the basal half areas in each case is shown. E. Evaluation of vessel size in the luminal and basal half areas of Non-n $(n=10)$, LGD $(n=7)$, flat-type HGD $(n=5)$ and sessile HGD lesions $(n=3)$. The ratio of average vessel density in the luminal half areas to that in the basal half areas in each case is shown. A Mann-Whitney $U$ test was performed. ${ }^{*} P<0.05$.

dysplasias, even with magnifying observation [20]. Our study used non-magnified white-color endoscopy to analyze the gross vascular effect, rather than the single vessel architecture or magnified surface pattern; all flat dysplastic lesions (including LGDs) were recognized as localized red-colored areas.

The relationship of the microvascular architecture and cancer has been examined in various gastrointestinal tumors; an irregular vasculature pattern and heterogeneity, as well as microvascular dilatation, have been reported [10,21, 22]. NBI enables detailed observation of capillaries on the mucosal surface, due to emission of narrow-band light, and can be used for qualitative diagnosis [23]. Based on a meta-analysis of four randomized trials regarding detection of colitis-associated dysplasia, NBI is not suggested in place of chromoendoscopy because it lacks any meaningful benefit $[3,24,25]$. However, these studies did not use magnified endoscopy for evaluation, and the diversity of inflamed background mucosa in patients with UC may have contributed to bias in the results. There are greater numbers of intramucosal vessels under neoplastic and inflammatory conditions [26,27]; our histological findings supported these prior data, in that the vessel density in non-neoplastic areas differed considerably among patients in the present study (Supplementary Table 3). However, the ratio of microvascular density/size was significantly greater in dysplastic lesions than in adjacent non-neoplastic lesions in all cases, suggesting the need for surveillance of enhanced reddish areas in inflamed mucosa. Moreover, the vascular-increased area was restricted to dysplastic lesions, which indicates that detection of reddish areas with a distinct margin by magnified NBI is a marker of flattype dysplasia. Although these endoscopic findings can facilitate the detection of flat-type dysplasias in patients with UC, accurate identification of these lesions in severely inflamed and regenerative mucosa remains problematic. In our patients, all flat-type dysplastic lesions were detected during clinical remission; thus, less severe inflammation is related to the improved detection of flat-type dysplasias in patients with UC.

The progression of inflamed mucosa in UC is generally assumed to proceed from colitis without dysplasia to LGD, then 


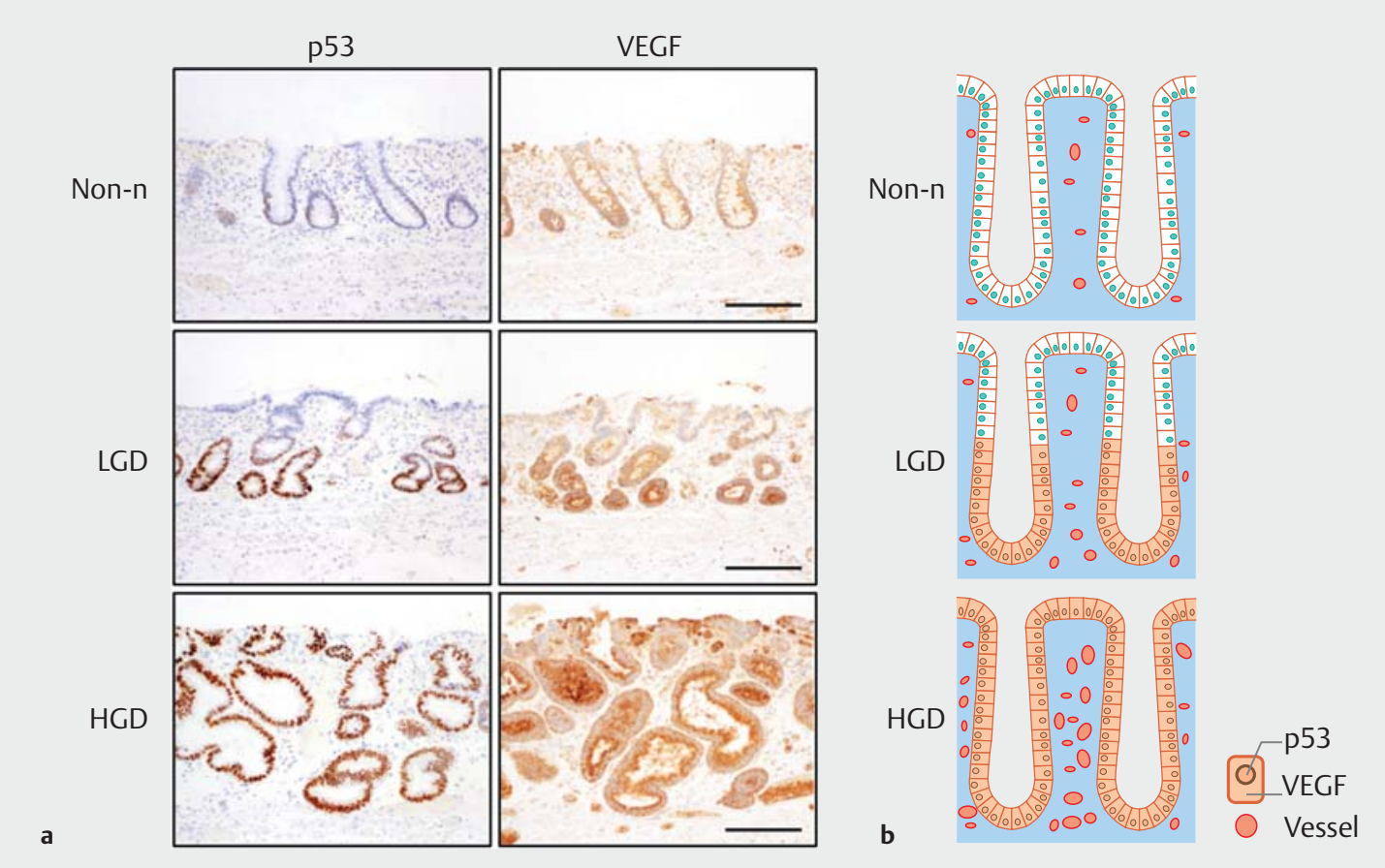

- Fig. 5 Increased expression of vascular endothelial growth factor in dysplastic epithelia. a Representative images of p53 and vascular endothelial growth factor (VEGF) expression in non-neoplastic (Non-n), flat-type low-grade dysplasia (LGD) and flat-type high-grade dysplasia (HGD) (Case \#4). Note that flat-type LGD and HGD epithelia expressed increased expression of VEGF compared to the surrounding non-neoplastic epithelia. Moreover, the pattern of VEGF expression in dysplastic epithelia was similar to that of p53. Scale bars, $200 \mu \mathrm{m}$. b Schematic presentation showing the association among epithelial p53 expression, VEGF expression and stromal vascular alteration.

HGD, and ultimately carcinoma. In colitis-associated carcinogenesis, p53 alterations occur early (i.e., before dysplasia has developed), in contrast to the disease course in sporadic neoplasia [2]. Histologically, LGDs contain p53-altered atypical epithelia confined largely to the basal half of the gland, whereas atypical epithelia extend into the superficial part of the gland in HGDs [15]. Notably, we found that increased/enlarged vessels were mainly present around p53-positive atypical epithelia in dysplastic glands ( Fig.2a and $>$ Fig.3), which indicated that TP53 mutations induce angiogenesis in UC-associated dysplasia. Ravi et al. demonstrated that the loss of p53 in tumor cells enhances the hypoxia-inducible factor- $1 \alpha$ level and augments hypoxia-inducible factor- $1 \alpha$-dependent transcriptional activation of VEGF [28]. A recent study using data from 7,525 tumor samples in the TCGA database revealed that the VEGFA mRNA level was significantly increased in TP53-mutated adenocarcinomas, compared to TP53 wild-type adenocarcinomas [29]. In the present study, the expression pattern of VEGF in dysplastic epithelia was similar to the expression pattern of p53, indicating links among TP53 alterations, VEGF expression, and stromal vascular changes ( $>$ Fig. $\mathbf{5 c}$ ). In this study, both LGD and HGD lesions were recognized as demarcated red-colored lesions and the intramucosal vascular densities/sizes were not significantly different among flat LGD, flat HGD and sessile HGD. However, considering the distribution of increased/enlarged vessels in LGDs and HGDs and given the depth reached by endoscopic light, the detection of LGDs may be more difficult than the detection of HGDs. Development of endoscopic technologies, such as linked color imaging, which provides better color contrast than white-light imaging, may be useful for visualization of LGDs [30].

This study had several limitations. First, it included a small number of patients and lesions from a single institution. Although a larger number of patients must be evaluated, flattype dysplasia was formerly recognized as invisible dysplasia; therefore, few flat-type dysplasias have been detected endoscopically. In this study, we analyzed totally resected flat-type dysplasias with available detailed endoscopic information, which is an advantage relative to other studies of flat-type dysplasias detected by random biopsy without endoscopic images. Second, although rare, colorectal signet-ring cell carcinomas occur more frequently in patients with UC. Our preliminary study revealed that signet-ring cell carcinomas were endoscopically recognized as discolored areas and histologically contained fewer vessels, suggesting the association between undifferentiated carcinoma lesions and less vascular densities. Moreover, it remains unclear whether all flat-type dysplasias other than signet-ring cell carcinoma can be identified as a reddish area. To evaluate the clinical utility of our findings, further studies are needed to examine flat-type dysplasias that could not be detected by endoscopy and were unexpectedly identified in resected specimens. Third, the "demarcated red-colored change," a key finding of this study, was only evaluated using endoscopic images; no further digital evaluations were performed. Despite these limitations, our endoscopic and histolo- 

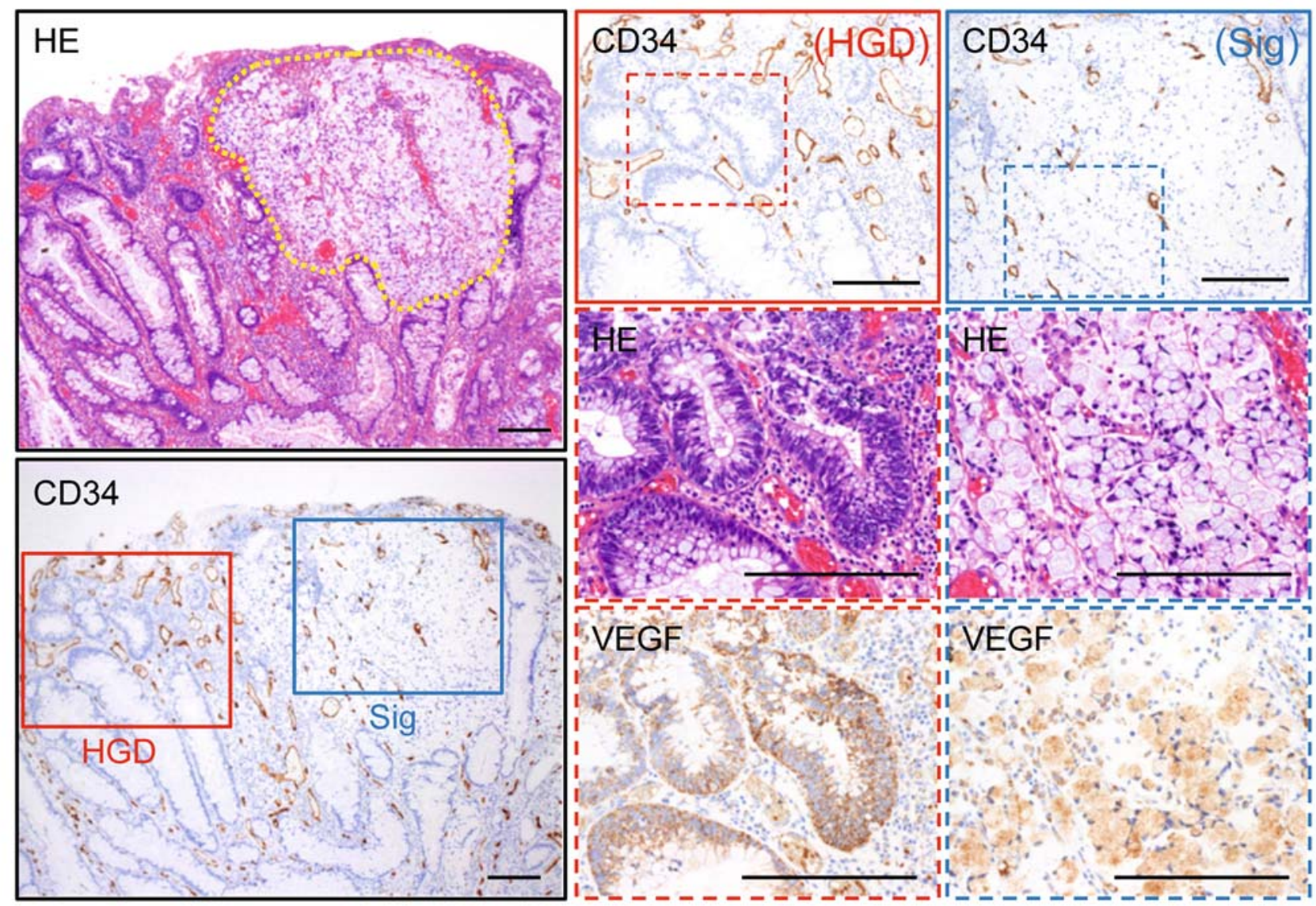

Fig. 6 Less vascular density/size in intramucosal signet-ring cell carcinoma areas. Representative histological images of colitis-associated tumor containing intramucosal high-grade dysplasia (HGD) and signet-ring cell carcinoma (Sig) components (Case \#S1). HE staining (upper left) shows intramucosal HGD containing a Sig component which is lined by a yellow dotted line. CD34 staining indicates intramucosal vessels in these lesions (lower left). The rectangle HGD (red line) and Sig (blue line) areas in the CD34 staining are magnified to the upper right. Further magnified HE staining and VEGF staining were shown below. Scale bars, $200 \mu \mathrm{m}$.

gical analyses can enhance surveillance colonoscopy for flattype dysplasias in patients with UC.

\section{Conclusion}

In conclusion, to the best of our knowledge, this study provides the first evidence of the endoscopic and histological features of flat-type dysplasia in patients with UC. We emphasize that the endoscopic recognition of "demarcated red-colored change" associated with vascular alteration is a marker of flat-type dysplasia and an indicator of the need for targeted biopsy. Identification of reddish areas with a distinct margin by magnifying NBI would enable clinicians to distinguish flat-type dysplasia from non-neoplastic inflamed mucosa.

\section{Competing interests}

The authors declare that they have no conflict of interest.
References

[1] Jess T, Rungoe C, Peyrin-Biroulet L. Risk of colorectal cancer in patients with ulcerative colitis: a meta-analysis of population-based cohort studies. Clin Gastroenterol Hepatol 2012; 10: 639-645

[2] Eaden JA, Abrams KR, Mayberry JF. The risk of colorectal cancer in ulcerative colitis: a meta-analysis. Gut 2001; 48: 526-535

[3] Laine L, Kaltenbach T, Barkun A et al. SCENIC international consensus statement on surveillance and management of dysplasia in inflammatory bowel disease. Gastrointest Endosc 2015; 81: 489-501.e26

[4] Laine L, Kaltenbach T, Barkun A et al. SCENIC international consensus statement on surveillance and management of dysplasia in inflammatory bowel disease. Gastroenterology 2015; 148: 639-651 e28

[5] Sugimoto S, Naganuma M, Iwao Y et al. Endoscopic morphologic features of ulcerative colitis-associated dysplasia classified according to the SCENIC consensus statement. Gastrointest Endosc 2017; 85: 639-646.e2

[6] Farraye FA, Odze RD, Eaden J et al. AGA technical review on the diagnosis and management of colorectal neoplasia in inflammatory bowel disease. Gastroenterology 2010; 138: 746-774, 74 e1-4; quiz e12-3 
[7] Van Assche G, Dignass A, Bokemeyer B et al. Second European evidence-based consensus on the diagnosis and management of ulcerative colitis part 3: special situations. J Crohn Colitis 2013; 7: 1-33

[8] Honmyo U, Misumi A, Murakami A et al. Mechanisms producing color change in flat early gastric cancers. Endoscopy 1997; 29: 366-371

[9] Kawamura M, Naganuma $H$, Shibuya R et al. Analysis of microvascular density in early gastric carcinoma using magnifying endoscopy with narrow-band imaging. Endosc Int Open 2016; 4: E832-E837

[10] Ohashi A, Niwa Y, Ohmiya N et al. Quantitative analysis of the microvascular architecture observed on magnification endoscopy in cancerous and benign gastric lesions. Endoscopy 2005; 37: 1215-1219

[11] Kinoshita S, Uraoka T, Nishizawa T et al. The role of colorectal endoscopic submucosal dissection in patients with ulcerative colitis. Gastrointest Endosc 2018; 87: 1079-1084

[12] Sugimoto S, Shimoda M, Iwao Y et al. Intramucosal poorly differentiated and signet-ring cell components in patients with ulcerative colitis-associated high-grade dysplasia. Digest Endosc 2019; 31: 706711

[13] Shima T, Shimoda M, Shigenobu T et al. Infiltration of tumor-associated macrophages is involved in tumor programmed death-ligand 1 expression in early lung adenocarcinoma. Cancer Sci 2020; 111: 727738

[14] Shimizu H, Shimoda M, Mochizuki S et al. Hyaluronan-binding protein involved in hyaluronan depolymerization is up-regulated and involved in hyaluronan degradation in human osteoarthritic cartilage. Am J Pathology 2018; 188: 2109-2019

[15] Riddell RH, Goldman H, Ransohoff DF et al. Dysplasia in inflammatory bowel disease : standardized classification with provisional clinical applications. Human Pathology 1983; 14: 931-968

[16] Apte RS, Chen DS, Ferrara N. VEGF in signaling and disease : beyond discovery and development. Cell 2019; 176: 1248-1264

[17] Chu PG, Weiss LM. Immunohistochemical characterization of signetring cell carcinomas of the stomach, breast, and colon. Am J Clin Pathology 2004; 121: 884-892

[18] Watanabe T, Ajioka Y, Mitsuyama K et al. Comparison of targeted vs random biopsies for surveillance of ulcerative colitis-associated colorectal cancer. Gastroenterology 2016; 151: 1122-1130
[19] Kawasaki K, Nakamura S, Esaki M et al. Clinical usefulness of magnifying colonoscopy for the diagnosis of ulcerative colitis-associated neoplasia. Digest Endosc 2019; 31: 36-42

[20] Shinagawa T, Hata K, Morikawa T et al. Pine-cone and villi patterns are endoscopic signs suggestive of ulcerative colitis-associated colorectal cancer and dysplasia. Gastrointest Endosc 2019; 89: 565-575 e3

[21] Kanesaka T, Uedo N, Yao K et al. A significant feature of microvessels in magnifying narrow-band imaging for diagnosis of early gastric cancer. Endosc Int Open 2015; 3: E590-E596

[22] Kaise M, Kato M, Urashima M et al. Magnifying endoscopy combined with narrow-band imaging for differential diagnosis of superficial depressed gastric lesions. Endoscopy 2009; 41: 310-315

[23] Gono K, Obi T, Yamaguchi M et al. Appearance of enhanced tissue features in narrow-band endoscopic imaging. J Biomed Optics 2004; 9: 568-577

[24] Efthymiou M, Allen PB, Taylor AC et al. Chromoendoscopy versus narrow band imaging for colonic surveillance in inflammatory bowel disease. Inflamm Bowel Dis 2013; 19: 2132-2138

[25] Pellise M, Lopez-Ceron M, Rodriguez de Miguel C et al. Narrow-band imaging as an alternative to chromoendoscopy for the detection of dysplasia in long-standing inflammatory bowel disease: a prospective, randomized, crossover study. Gastrointest Endosc 2011; 74: $840-848$

[26] Scaldaferri F, Vetrano S, Sans M et al. VEGF-A links angiogenesis and inflammation in inflammatory bowel disease pathogenesis. Gastroenterology 2009; 136: 585-595 e5

[27] Chung AS, Ferrara N. Developmental and pathological angiogenesis. Ann Rev Cell Develop Biology 2011; 27: 563-584

[28] Ravi R, Mookerjee B, Bhujwalla ZM et al. Regulation of tumor angiogenesis by $\mathrm{p} 53$-induced degradation of hypoxia-inducible factor 1alpha. Genes Develop 2000; 14: 34-44

[29] Li AM, Boichard A, Kurzrock R. Mutated TP53 is a marker of increased VEGF expression: analysis of 7,525 pan-cancer tissues. Cancer Biol Ther 2020; 21: 95-100

[30] Kanzaki H, Takenaka R, Kawahara Y et al. Linked color imaging (LCI), a novel image-enhanced endoscopy technology, emphasizes the color of early gastric cancer. Endosc Int Open 2017; 5: E1005-E1013 